\title{
Article
}

\section{Joint Universality of the Zeta-Functions of Cusp Forms}

\section{Renata Macaitiené (D)}

check for updates

Citation: Macaitienè, R. Joint Universality of the Zeta-Functions of Cusp Forms. Mathematics 2021, 9 , 2161. https://doi.org/10.3390/ math9172161

Academic Editor: Yang-Hui He

Received: 26 July 2021

Accepted: 31 August 2021

Published: 4 September 2021

Publisher's Note: MDPI stays neutral with regard to jurisdictional claims in published maps and institutional affiliations.

Copyright: (c) 2021 by the authors. Licensee MDPI, Basel, Switzerland. This article is an open access article distributed under the terms and conditions of the Creative Commons Attribution (CC BY) license (https:// creativecommons.org/licenses/by/ $4.0 /)$.
Regional Development Institute of Šiauliai Academy, Vilnius University, P. Višinskio Str. 25, LT-76351 Šiauliai, Lithuania; renata.macaitiene@sa.vu.lt

Abstract: Let $F$ be the normalized Hecke-eigen cusp form for the full modular group and $\zeta(s, F)$ be the corresponding zeta-function. In the paper, the joint universality theorem on the approximation of a collection of analytic functions by shifts $\left(\zeta\left(s+i h_{1} \tau, F\right), \ldots, \zeta\left(s+i h_{r} \tau, F\right)\right)$ is proved. Here, $h_{1}, \ldots, h_{r}$ are algebraic numbers linearly independent over the field of rational numbers.

Keywords: Hecke-eigen cusp form; joint universality; universality; zeta-function

MSC: 11M46

\section{Introduction}

The series of the types

$$
\sum_{m=1}^{\infty} \frac{a_{m}}{m^{s}} \text { and } \sum_{m=1}^{\infty} a_{m} \mathrm{e}^{-\lambda_{m} s}, \quad s=\sigma+i t,
$$

where $\left\{\lambda_{m}\right\}$ is a nondecreasing sequence of real numbers and $\lim _{m \rightarrow \infty} \lambda_{m}=+\infty$ are called Dirichlet series. The majority of zeta-functions are meromorphic functions in some halfplane defined by Dirichlet series having a certain arithmetic sense. The most important of zeta-functions is the Riemann zeta-function

$$
\zeta(s)=\sum_{m=1}^{\infty} \frac{1}{m^{s}}, \quad \sigma>1 .
$$

In [1], Voronin discovered a very interesting and important property of $\zeta(s)$ to approximate a wide class of analytic functions by shifts $\zeta(s+i \tau), \tau \in \mathbb{R}$, and called it universality. Later, it turned out that some other zeta-functions also are universal in the Voronin sense. This paper is devoted to the universality of zeta-functions of certain cusp forms.

Let

$$
S L(2, \mathbb{Z})=\left\{\left(\begin{array}{ll}
a & b \\
c & d
\end{array}\right): a, b, c, d \in \mathbb{Z}, a d-b c=1\right\}
$$

be the full modular group. If the function $F(z)$ is holomorphic in the upper half-plane $\operatorname{Im} z>0$, and for all elements of $S L(2, \mathbb{Z})$ with some $\kappa \in 2 \mathbb{N}$ satisfies the functional equation

$$
F\left(\frac{a z+b}{c z+d}\right)=(c z+d)^{\kappa} F(z)
$$

where $F(z)$ is called a modular form of weight $\kappa$ for the full modular group. Then, $F(z)$ has Fourier series expansion

$$
F(z)=\sum_{m=-\infty}^{\infty} c(m) \mathrm{e}^{2 \pi i m z}
$$


If $c(m)=0$ for all $m \leqslant 0$, then $F(z)$ is a cusp form of weight $\kappa$. The corresponding zeta-function (or $L$-function) $\zeta(s, F)$ is defined for $\sigma>\frac{\kappa+1}{2}$ by the Dirichlet series

$$
\zeta(s, F)=\sum_{m=1}^{\infty} \frac{c(m)}{m^{s}}
$$

and has the analytic continuation to an entire function. Additionally, we suppose that $F(z)$ is a simultaneous eigenfunction of all Hecke operators $T_{m}$

$$
T_{m} F(z)=m^{\kappa-1} \sum_{\substack{a, d>0 \\ a d=m}} \frac{1}{d^{\kappa}} \sum_{b(\bmod d)} F\left(\frac{a z+b}{d}\right), \quad m \in \mathbb{N} .
$$

In this case, $c(1) \neq 0$; therefore, the form $F(z)$ can be normalized, and thus, we may suppose that $c(1)=1$.

Now, we suppose that $F(z)$ is a normalized Hecke-eigen cusp form of weight $\kappa$ for the full modular group. Then, the zeta-function $\zeta(s, F)$ can be written, for $\sigma>\frac{\kappa+1}{2}$, as a product over primes

$$
\zeta(s, F)=\prod_{p}\left(1-\frac{\alpha(p)}{p^{s}}\right)^{-1}\left(1-\frac{\beta(p)}{p^{s}}\right)^{-1},
$$

where $\alpha(p)$ and $\beta(p)$ are conjugate complex numbers satisfying the equality $\alpha(p)+\beta(p)=$ $c(p)$.

In the paper [2], the universality of the function $\zeta(s, F)$ was proved. Let $D_{\kappa}=\{s \in$ $\left.\mathbb{C}: \frac{\kappa}{2}<\sigma<\frac{\kappa+1}{2}\right\}, \mathcal{K}_{F}$ be the class of compact subsets of the strip $D_{\kappa}$ with connected complements, and $H_{0, F}(K), K \in \mathcal{K}_{F}$ the class of continuous nonvanishing functions on $K$ that are analytic in the interior of $K$. Moreover, let meas $A$ denote the Lebesgue measure of a measurable set $A \subset \mathbb{R}$. Then, in [2], the following theorem was obtained.

Theorem 1. Suppose that $K \in \mathcal{K}_{F}$ and $f(s) \in H_{0, F}(K)$. Then, for every $\varepsilon>0$,

$$
\liminf _{T \rightarrow \infty} \frac{1}{T} \text { meas }\left\{\tau \in[0, T]: \sup _{s \in K}|\zeta(s+i \tau, F)-f(s)|<\varepsilon\right\}>0 .
$$

Theorem 1 shows that there are infinitely many shifts $\zeta(s+i \tau, F)$ approximating a given function $f(s) \in H_{0, F}$. In the shifts $\zeta(s+i \tau, F)$ of Theorem $1, \tau$ takes arbitrary real values; therefore, the theorem is of continuous type. Further, discrete universality theorems for the function $\zeta(s, F)$ are known. In [3,4], the discrete universality theorems with shifts $\zeta(s+i k h, F), k \in \mathbb{N}, h>0$ being a fixed number, were proved. Denote by $H\left(D_{\kappa}\right)$ the space of analytic on $D_{\kappa}$ functions endowed with the topology of uniform convergence on compacta. The paper [5] is devoted to the universality for compositions $\Phi(\zeta(s, F))$ with certain operators $\Phi: H\left(D_{\kappa}\right) \rightarrow H\left(D_{\kappa}\right)$. The results of the latter paper were applied in [6] for the functional independence of the compositions $\Phi(\zeta(s, F))$.

Let, for a fixed $l \in \mathbb{N}$,

$$
\Gamma_{0}(l)=\left\{\left(\begin{array}{ll}
a & b \\
c & d
\end{array}\right) \in S L(2, \mathbb{Z}): c \equiv 0(\bmod l)\right\}
$$

denote the Hecke subgroup of the group $S L(2, \mathbb{Z})$. If $F(z)$ satisfies (1) for all elements of $\Gamma_{0}(l)$, then $F(z)$ is called a cusp form of weight $\kappa$ and level $l$. The form $F(z)$ is called a new form if it is not a cusp form of level $l_{1} \mid l$. In [7], a universality theorem was obtained for zeta-functions of new forms.

The universality theorem of [2] was generalized in [8] for shifts $\zeta(s+i \varphi(\tau), F)$ with differentiable function $\varphi(\tau)$ satisfying the estimates $\left(\varphi^{\prime}(\tau)\right)^{-1}=o(\tau)$ and $\varphi(2 \tau) \max _{\tau \leqslant t \leqslant 2 \tau}\left(\varphi^{\prime}(t)\right)^{-1}$ 
$\ll \tau$ as $\tau \rightarrow \infty$. The discrete version of results of [8] is given in [9]. In [10], the shifts $\zeta\left(s+i \gamma_{k}, F\right)$, where $\left\{\gamma_{k}: k \in \mathbb{N}\right\}$ is the sequence of nontrivial zeros of $\zeta(s)$, are used.

The joint universality of zeta- and $L$-functions is a more complicated problem of analytic number theory. In this case, a collection of analytic functions are simultaneously approximated by a collection of shifts of zeta-functions. The first result in this direction also belongs to Voronin. He considered [11] the functional independence of Dirichlet $L$-functions $L(s, \chi)$ with pairwise nonequivalent Dirichlet characters $\chi$ and, for this, he obtained their joint universality. The paper [12] is devoted to the joint universality for zeta-functions of new forms twisted by Dirichlet characters, i.e., for the functions

$$
\sum_{m=1}^{\infty} \frac{c(m) \chi(m)}{m^{s}}, \quad \sigma>\frac{\kappa+1}{2}
$$

with pairwise nonequivalent Dirichlet characters $\chi_{1}, \ldots, \chi_{r}$.

Joint universality theorems with generalized shifts $\zeta\left(s+i \varphi_{j}(k), F\right), j=1, \ldots, r$, with some differentiable functions $\varphi_{j}(\tau)$ can be found in [13]. Continuous and discrete joint universality theorems for more general zeta-functions are given in [14-16].

Our aim is to obtain a joint universality theorem for zeta-functions of normalized Hecke-eigen cusp forms by using different shifts. The first of the denseness results for shifts of a universal function were discussed in [17].

The main result of the paper is the following statement.

Theorem 2. Suppose that $h_{1}, \ldots, h_{r}$ are real algebraic numbers linearly independent over the field of rational numbers $\mathbb{Q}$. For $j=1, \ldots, r$, let $K_{j} \in \mathcal{K}_{F}$ and $f_{j}(s) \in H_{0, F}\left(K_{j}\right)$. Then, for every $\varepsilon>0$,

$$
\liminf _{T \rightarrow \infty} \frac{1}{T} \text { meas }\left\{\tau \in[0, T]: \sup _{1 \leqslant j \leqslant r} \sup _{s \in K_{j}}\left|\zeta\left(s+i h_{j} \tau, F\right)-f_{j}(s)\right|<\varepsilon\right\}>0 .
$$

Moreover "lim inf" can be replaced by "lim" for all but at most countably many $\varepsilon>0$.

For the proof of Theorem 2, we will apply the probabilistic approach based on a limit theorem in the space of analytic functions.

\section{Mean Square Estimates}

Recall the metric in the space $H\left(D_{\kappa}\right)$. Let $\left\{K_{l}: l \in \mathbb{N}\right\} \subset D_{\mathcal{K}}$ be a sequence of compact subsets such that

$$
D_{\kappa}=\bigcup_{l=1}^{\infty} K_{l}
$$

$K_{l} \subset K_{l+1}$ for $l \in \mathbb{N}$, and if $K \subset D_{\kappa}$ is a compact, then $K \subset K_{l}$ for some $l$. For example, we can take $K_{l}$ closed rectangles. Then

$$
\rho\left(g_{1}, g_{2}\right)=\sum_{l=1}^{\infty} 2^{-l} \frac{\sup _{s \in K_{l}}\left|g_{1}(s)-g_{2}(s)\right|}{1+\sup _{s \in K_{l}}\left|g_{1}(s)-g_{2}(s)\right|}, \quad g_{1}, g_{2} \in H\left(D_{\kappa}\right),
$$

is a metric in $H\left(D_{\kappa}\right)$ inducing the topology of uniform convergence on compacta.

Let

$$
H^{r}\left(D_{\kappa}\right)=(\underbrace{H\left(D_{\kappa}\right) \times \cdots \times H\left(D_{\kappa}\right)}_{r} .
$$

For $\underline{g}_{j}=\left(g_{j 1}, \ldots, g_{j r}\right) \in H^{r}\left(D_{\kappa}\right), j=1,2$, define

$$
\underline{\rho}\left(\underline{g}_{1}, \underline{g}_{2}\right)=\max _{1 \leqslant j \leqslant r} \rho\left(g_{1 j}, g_{2 j}\right) .
$$


Then, $\rho$ is a metric in $H^{r}\left(D_{\kappa}\right)$ inducing the product topology.

Let $\theta>\frac{1}{2}$ be a fixed number, and

$$
v_{n}(m)=\exp \left\{-\left(\frac{m}{n}\right)^{\theta}\right\}, \quad m, n \in \mathbb{N} .
$$

Then, the series

$$
\zeta_{n}(s, F)=\sum_{m=1}^{\infty} \frac{c(m) v_{n}(m)}{m^{s}},
$$

in view of the estimate

$$
c(m) \ll m^{\frac{\kappa-1}{2}+\varepsilon},
$$

is absolutely convergent in every fixed half plane $\sigma>\hat{\sigma}$. However, for our aim, this convergence is sufficient only for $\sigma>\frac{\kappa}{2}$.

For brevity, let $\underline{h}=\left(h_{1}, \ldots, h_{r}\right)$,

$$
\underline{\zeta}(s+i \underline{h} \tau, F)=\left(\zeta\left(s+i h_{1} \tau, F\right), \ldots, \zeta\left(s+i h_{r} \tau, F\right)\right)
$$

and

$$
\underline{\zeta}_{n}(s+i \underline{h} \tau, F)=\left(\zeta_{n}\left(s+i h_{1} \tau, F\right), \ldots, \zeta_{n}\left(s+i h_{r} \tau, F\right)\right) .
$$

Lemma 1. For all $h$

$$
\lim _{n \rightarrow \infty} \limsup _{T \rightarrow \infty} \frac{1}{T} \int_{0}^{T} \underline{\rho}\left(\underline{\zeta}(s+i \underline{h} \tau, F), \underline{\zeta}_{n}(s+i \underline{h} \tau, F)\right) \mathrm{d} \tau=0 .
$$

Proof. By the definitions of the metrics $\rho$ and $\underline{\rho}$, it suffices to show that, for every $h \in \mathbb{R}$ and compact set $K \subset D_{K}$,

$$
\left.\lim _{n \rightarrow \infty} \limsup _{T \rightarrow \infty} \frac{1}{T} \int_{0}^{T} \sup _{s \in K} \mid \zeta(s+i h \tau, F), \zeta_{n}(s+i h \tau, F)\right) \mathrm{d} \tau=0 .
$$

It is well known that for fixed $\frac{\kappa}{2}<\sigma<\frac{\kappa+1}{2}$,

$$
\int_{-T}^{T}|\zeta(\sigma+i t, F)|^{2} \mathrm{~d} t \ll_{\sigma} T,
$$

where $\ll_{\sigma}$ means that the implied constant depends on $\sigma$. Therefore,

$$
\int_{-T}^{T}|\zeta(\sigma+i h t, F)|^{2} \mathrm{~d} t \ll_{\sigma, h} T
$$

and, for $v \in \mathbb{R}$,

$$
\frac{1}{T} \int_{0}^{T}|\zeta(\sigma+i h \tau+i v, F)|^{2} \mathrm{~d} v \ll_{\sigma, h} 1+|v|
$$

Let

$$
l_{n}(s)=\frac{z}{\theta} \Gamma\left(\frac{z}{\theta}\right) n^{z},
$$


where $\Gamma(z)$ denotes the Euler gamma-function and $\theta$ is a number from the definition of $v_{n}(m)$. Using the Mellin formula

$$
\frac{1}{2 \pi i} \int_{\beta-i \infty}^{\beta+i \infty} \Gamma(s) \alpha^{s} \mathrm{~d} s=\mathrm{e}^{-\alpha}, \quad \alpha, \beta>0,
$$

we find that

$$
\exp \left\{-\left(\frac{m}{n}\right)^{\theta}\right\}=\frac{1}{2 \pi i} \int_{\theta-i \infty}^{\theta+i \infty} \frac{1}{\theta} \Gamma\left(\frac{1}{\theta}\right)\left(\frac{m}{n}\right)^{-s} \mathrm{~d} s .
$$

Therefore, in virtue of the definition of the function $v_{n}(m)$, we obtain that, for $\sigma>\frac{\kappa}{2}$,

$$
\begin{aligned}
\zeta_{n}(s, F) & =\frac{1}{2 \pi i} \sum_{m=1}^{\infty} \frac{c(m)}{m^{s}} \int_{\theta-i \infty}^{\theta+i \infty} \frac{z}{\theta} \Gamma\left(\frac{z}{\theta}\right)\left(\frac{m}{n}\right)^{-z} \frac{\mathrm{d} z}{z} \\
& =\frac{1}{2 \pi i} \int_{\theta-i \infty}^{\theta+i \infty}\left(\frac{l_{n}(z)}{z} \sum_{m=1}^{\infty} \frac{c(m)}{m^{s+z}}\right) \mathrm{d} z \\
& =\frac{1}{2 \pi i} \int_{\theta-i \infty}^{\theta+i \infty} \zeta(s+z, F) l_{n}(z) \frac{\mathrm{d} z}{z} .
\end{aligned}
$$

Let $K \in D_{\mathcal{K}}$ be a fixed compact set. Then, there exists $\varepsilon>0$ such that, for all $s=$ $\sigma+i t \in K$, the inequalities $\frac{\kappa}{2}+2 \varepsilon<\sigma<\frac{\kappa+1}{2}-\varepsilon$ are satisfied. We take, for such $\sigma$,

$$
\theta_{1}=\frac{\kappa}{2}+\varepsilon-\sigma
$$

Then, $\theta_{1}<0$. Therefore, by the residue theorem and (3),

$$
\zeta_{n}(s, F)-\zeta(s, F)=\frac{1}{2 \pi i} \int_{\theta_{1}-i \infty}^{\theta_{1}+i \infty} \zeta(s+z, F) l_{n}(z) \frac{\mathrm{d} z}{z} .
$$

Hence, for all $s \in K$,

$$
\begin{aligned}
\zeta(s+i h \tau, F)-\zeta_{n}(s+i h \tau, F) & =\frac{1}{2 \pi i} \int_{-\infty}^{\infty} \zeta\left(\frac{\kappa}{2}+\varepsilon+i t+i h \tau+i v, F\right) \frac{\ln \left(\frac{\kappa}{2}+\varepsilon-\sigma+i v\right)}{\frac{\kappa}{2}+\varepsilon-\sigma+i v} \mathrm{~d} v \\
& =\frac{1}{2 \pi i} \int_{-\infty}^{\infty} \zeta\left(\frac{\kappa}{2}+\varepsilon+i h \tau+i v, F\right) \frac{\ln \left(\frac{\kappa}{2}+\varepsilon-s+i v\right)}{\frac{\kappa}{2}+\varepsilon-s+i v} \mathrm{~d} v \\
& \ll \frac{1}{2 \pi i} \int_{-\infty}^{\infty}\left|\zeta\left(\frac{\kappa}{2}+\varepsilon+i h \tau+i v, F\right)\right| \sup _{s \in K}\left|\frac{\ln \left(\frac{\kappa}{2}+\varepsilon-s+i v\right)}{\frac{\kappa}{2}+\varepsilon-s+i v}\right| \mathrm{d} v
\end{aligned}
$$

Thus, in view of (2),

$$
\begin{aligned}
& \frac{1}{T} \int_{0}^{\infty} \sup _{s \in K}\left|\zeta(s+i h \tau, F)-\zeta_{n}(s+i h \tau, F)\right| \mathrm{d} \tau \\
& \ll \int_{-\infty}^{\infty}\left(\left(\frac{1}{T} \int_{0}^{\infty}\left|\zeta\left(\frac{\kappa}{2}+\varepsilon+i h \tau+i v\right)\right|^{2} \mathrm{~d} \tau\right)^{1 / 2} \sup _{s \in K}\left|\frac{l_{n}\left(\frac{\kappa}{2}+\varepsilon-s+i v\right)}{\frac{\kappa}{2}+\varepsilon-s+i v}\right|\right) \mathrm{d} v
\end{aligned}
$$




$$
\ll_{\varepsilon, h, K} n^{-\varepsilon} \int_{-\infty}^{\infty}(1+|v|) \exp \left\{-c_{1}|v|\right\} \mathrm{d} v \ll_{\varepsilon, h, K} n^{-\varepsilon}
$$

Here, we used the estimate

$$
\Gamma\left(\frac{1}{\theta}\left(\frac{\kappa}{2}+\varepsilon-s+i v\right)\right) \ll \exp \left\{-\frac{c}{\theta}|v-t|\right\} \ll_{\kappa} \exp \left\{-c_{1}|v|\right\}, \quad c_{1}>0 .
$$

Estimate (4) proves the lemma.

Let $\mathbb{P}$ be the set of all prime numbers, and $\gamma_{p}=\{s \in \mathbb{C}:|s|=1\}$ for all $p \in \mathbb{P}$. Define the set

$$
\Omega=\prod_{p \in \mathbb{P}} \gamma_{p}
$$

Then, the torus $\Omega$ with product topology and pointwise multiplication is a compact topological Abelian group. Therefore, on $(\Omega, \mathcal{B}(\Omega))(\mathcal{B}(\mathbb{X})$ is the Borel $\sigma$-field of the space $\mathbb{X})$, the probability Haar measure $m_{H}$ can be defined. Moreover, let

$$
\underline{\Omega}=\Omega_{1} \times \cdots \times \Omega_{r}
$$

where $\Omega_{j}=\Omega$ for all $j=1, \ldots, r$. Once again, $\Omega$ is a compact topological Abelian group. Therefore, on $(\underline{\Omega}, \mathcal{B}(\underline{\Omega}))$ the probability Haar measure $\underline{m}_{H}$ exists. This gives the probability space $\left(\Omega, \mathcal{B}(\underline{\Omega}), \underline{m}_{H}\right)$. Denote by $m_{j H}$ the Haar measure on $\left(\Omega_{j}, \mathcal{B}\left(\Omega_{j}\right)\right), j=1, \ldots, r$. Then, $\underline{m}_{H}$ is the product of the measures $m_{1 H}, \ldots, m_{r H}$. Now, denote by $\underline{\omega}=\left(\omega_{1}, \ldots, \omega_{r}\right)$ the elements of $\underline{\Omega}$, where $\omega_{j} \in \Omega_{j}, j=1, \ldots, r$. Let $\omega_{j}(p)$ be the $p$ th component of an element $\omega_{j} \in \Omega_{j}, j=1, \ldots, r, p \in \mathbb{P}$. Extend elements $\omega_{j}(p)$ to the set $\mathbb{N}$ by the formula

$$
\omega_{j}(m)=\prod_{\substack{p^{l} \mid m \\ p^{l+1} \nmid m}} \omega_{j}^{l}(p), \quad m \in \mathbb{N}
$$

and define $H\left(D_{\kappa}\right)$-valued random element

$$
\zeta\left(s, \omega_{j}, F\right)=\sum_{m=1}^{\infty} \frac{c(m) \omega_{j}(m)}{m^{s}}, \quad j=1, \ldots, r .
$$

The later series is uniformly convergent on compact subsets of $D_{\kappa}$ for almost all $\omega_{j}$. Moreover, for fixed $\sigma \in\left(\frac{\kappa}{2}, \frac{\kappa+1}{2}\right)$

$$
\int_{-T}^{T}\left|\zeta\left(s+i t, \omega_{j}, F\right)\right|^{2} \mathrm{~d} t \ll_{\sigma} T
$$

for almost all $\omega_{j}, j=1, \ldots, r[18]$. Define one more series

$$
\zeta_{n}\left(s, \omega_{j}, F\right)=\sum_{m=1}^{\infty} \frac{c(m) \omega_{j}(m) v_{n}(m)}{m^{s}}, \quad j=1, \ldots, r,
$$

which also, as $\zeta_{n}(s, F)$, are absolutely convergent for $\sigma>\frac{\kappa}{2}$. Let

$$
\underline{\zeta}(s+i \underline{h} \tau, \underline{\omega}, F)=\left(\zeta\left(s+i h_{1} \tau, \omega_{1}, F\right), \ldots, \zeta\left(s+i h_{r} \tau, \omega_{1}, F\right)\right)
$$

and

$$
\underline{\zeta}_{n}(s+i \underline{h} \tau, \underline{\omega}, F)=\left(\zeta_{n}\left(s+i h_{1} \tau, \omega_{1}, F\right), \ldots, \zeta_{n}\left(s+i h_{r} \tau, \omega_{r}, F\right)\right) .
$$


Then, repeating the proof of Lemma 1 and using estimate (5), we arrive to the following statement.

Lemma 2. For all $\underline{h}$ and almost all $\underline{\omega}$,

$$
\lim _{n \rightarrow \infty} \limsup _{T \rightarrow \infty} \frac{1}{T} \int_{0}^{T} \underline{\rho}\left(\underline{\zeta}(s+i \underline{h} \tau, \underline{\omega}, F), \underline{\zeta}_{n}(s+\underline{i} \underline{h} \tau, \underline{\omega}, F)\right) \mathrm{d} \tau=0 .
$$

\section{Limit Theorems}

On the probability space $\left(\Omega, \mathcal{B}(\underline{\Omega}), \underline{m}_{H}\right)$, define $H\left(D_{\kappa}\right)$-valued random element

$$
\underline{\zeta}(s, \underline{\omega}, F)=\left(\zeta\left(s, \omega_{1}, F\right), \ldots, \zeta\left(s, \omega_{1}, F\right)\right)
$$

and denote by $P_{\underline{\underline{\zeta}}, F}$ its distribution, i.e.,

$$
P_{\underline{\zeta}, F}(A)=\underline{m}_{H}\{\underline{\omega} \in \underline{\Omega}: \underline{\zeta}(s, \underline{\omega}, F) \in A\}, \quad A \in \mathcal{B}\left(H^{r}\left(D_{\kappa}\right)\right) .
$$

Theorem 3. Suppose that $h_{1}, \ldots, h_{r}$ are real algebraic numbers linearly independent over $\mathbb{Q}$, and

$$
P_{T, F}(A) \stackrel{\text { def }}{=} \frac{1}{T} \text { meas }\{\tau \in[0, T]: \underline{\zeta}(s+i \underline{h} \tau, F) \in A\}, \quad A \in \mathcal{B}\left(H^{r}\left(D_{\kappa}\right)\right) .
$$

Then, $P_{T, F}$ converges weakly to $P_{\underline{\zeta}, F}$ as $T \rightarrow \infty$.

We divide the proof of Theorem 3 into several lemmas.

Lemma 3. Suppose that $\lambda_{1}, \ldots, \lambda_{r}$ are algebraic numbers such that the system $\log \lambda_{1}, \ldots, \log \lambda_{r}$ is linearly independent over $\mathbb{Q}$. Then, for arbitrary algebraic numbers $\beta_{0}, \beta_{1}, \ldots, \beta_{r}$ that are not all zeros, the inequality

$$
\left|\beta_{0}+\beta_{1} \log \lambda_{1}+\cdots+\beta_{r} \log \lambda_{r}\right|>h^{-c}
$$

holds. Here, $h$ denotes the height of the numbers $\beta_{0}, \beta_{1}, \ldots, \beta_{r}$, and $c$ is an effective constant depending on $r, \lambda_{1}, \ldots, \lambda_{r}$ and maximum of degrees of the numbers $\beta_{0}, \beta_{1}, \ldots, \beta_{r}$.

The lemma is a Baker result on linear forms of logarithm; see, for example, ref. [19]. For $A \in \mathcal{B}(\underline{\Omega})$, define

$$
Q_{T}(A)=\frac{1}{T} \operatorname{meas}\left\{\tau \in[0, T]:\left(\left(p^{-i h_{1} \tau}: p \in \mathbb{P}\right), \ldots,\left(p^{-i h_{r} \tau}: p \in \mathbb{P}\right)\right) \in A\right\} .
$$

Lemma 4. Let $\lambda_{1}, \ldots, \lambda_{r}$ be the same as in Theorem 3. Then, $Q_{T}$ converges weakly to the Haar measure $\underline{m}_{H}$ as $T \rightarrow \infty$.

Proof. We apply the Fourier transform method. Denote by $g_{T}\left(\underline{k}_{1}, \ldots, \underline{k}_{r}\right), \underline{k}_{j}=\left\{k_{p j}: k_{p j} \in\right.$ $\mathbb{Z}, p \in \mathbb{P}\}, j=1, \ldots, r$ the Fourier transform of $Q_{T}$. By the definition of $Q_{T}$, we have

$$
\begin{aligned}
g_{T}\left(\underline{k}_{1}, \ldots, \underline{k}_{r}\right)= & \int_{\underline{\Omega}} \prod_{j=1}^{r} \prod_{p \in \mathbb{P}}^{*} \omega_{j}^{k_{p j}}(p) \mathrm{d} Q_{T} \\
& \frac{1}{T} \int_{0}^{T} \exp \left\{-i \tau \sum_{j=1}^{k} \sum_{p \in \mathbb{P}}^{*} h_{j} k_{p j} \log p\right\} \mathrm{d} \tau,
\end{aligned}
$$

where the star shows that only a finite number of integers $k_{p j}$ are not zero. Obviously,

$$
g_{T}(\underline{0}, \ldots, \underline{0})=1 .
$$


Now, suppose that $\left(\underline{k}_{1}, \ldots, \underline{k}_{r}\right) \neq(\underline{0}, \ldots, \underline{0})$. Then, there exists a prime number $p$ such that $k_{p j} \neq 0$ for some $j$. Therefore,

$$
\beta_{p} \stackrel{\text { def }}{=} \sum_{j=1}^{r} h_{j} k_{p j} \neq 0
$$

because the numbers $h_{1}, \ldots, h_{r}$ are linearly independent over $Q$. Thus, in view of Lemma 3 ,

$$
B_{\underline{k}_{1}, \ldots, \underline{k}_{r}} \stackrel{\text { def }}{=} \sum_{j=1}^{k} \sum_{p \in \mathbb{P}}^{*} h_{j} k_{p j} \log p=\sum_{p \in \mathbb{P}}^{*} \beta_{p} \log p \neq 0 .
$$

This and (6) imply

$$
g_{T}\left(\underline{k}_{1}, \ldots, \underline{k}_{r}\right)=\frac{1-\exp \left\{-i T B_{\underline{k}_{1}, \ldots, \underline{k}_{r}}\right\}}{i T B_{\underline{k}_{1}, \ldots, \underline{k}_{r}}} .
$$

Therefore, by (7),

$$
\lim _{T \rightarrow \infty} g_{T}\left(\underline{k}_{1}, \ldots, \underline{k}_{r}\right) \stackrel{\text { def }}{=} \begin{cases}1 & \text { if }\left(\underline{k}_{1}, \ldots, \underline{k}_{r}\right)=(\underline{0}, \ldots, \underline{0}), \\ 0 & \text { if }\left(\underline{k}_{1}, \ldots, \underline{k}_{r}\right) \neq(\underline{0}, \ldots, \underline{\underline{0}}),\end{cases}
$$

and this proves the lemma.

For $A \in \mathcal{B}\left(H^{r}\left(D_{\mathcal{K}}\right)\right)$, define

$$
P_{T, n, F}(A)=\frac{1}{T} \operatorname{meas}\left\{\tau \in[0, T]: \underline{\zeta}_{n}(s+i \underline{h} \tau, F) \in A\right\}
$$

and

$$
P_{T, n, \underline{\Omega}, F}(A)=\frac{1}{T} \operatorname{meas}\left\{\tau \in[0, T]: \underline{\zeta}_{n}(s+i \underline{h} \tau, \underline{\omega}, F) \in A\right\} .
$$

Moreover, let the mapping $u_{n}: \underline{\Omega} \rightarrow H^{r}\left(D_{\kappa}\right)$ be given by

$$
u_{n, F}(\underline{\omega})=\underline{\zeta}_{n}(s, \underline{\omega}, F),
$$

and $V_{n, F}=\underline{m}_{H} u_{n, F}^{-1}$, where

$$
V_{n, F}(A)=\underline{m}_{H}\left(u_{n, F}^{-1} A\right), \quad A \in \mathcal{B}\left(H^{r}\left(D_{\kappa}\right)\right) .
$$

Since the series for $\zeta_{n}\left(s, \omega_{j}, F\right)$ are absolutely convergent for $\sigma>\frac{\kappa}{2}$, the mapping $u_{n, F}$ is continuous. Moreover, by the definitions of $Q_{T}$ and $P_{T, n, F}$, we have $P_{T, n, F}=Q_{T} u_{n, F}^{-1}$. This equality, continuity of $u_{n, F}$, Lemma 4 , the well-known properties of weak convergence, and the invariance of the Haar measure $\underline{m}_{H}$ lead to the following lemma.

Lemma 5. Let $h_{1}, \ldots, h_{r}$ be the same as Theorem 3. Then, $P_{T, n, F}$ and $P_{T, n, \Omega, F}$ both converge weakly to the measure $V_{n, F}$ as $T \rightarrow \infty$.

Additionally to $P_{T, F}$, define

$$
P_{T, \underline{\Omega}, F}(A)=\frac{1}{T} \operatorname{meas}\{\tau \in[0, T]: \underline{\zeta}(s+i \tau, \underline{\omega}, F) \in A\}, \quad A \in \mathcal{B}\left(H^{r}\left(D_{\kappa}\right)\right) .
$$

Lemma 6. Let $h_{1}, \ldots, h_{r}$ be the same as Theorem 3. Then, on $\left(H^{r}\left(D_{\kappa}\right), \mathcal{B}\left(H^{r}\left(D_{\kappa}\right)\right)\right)$, there exists a probability measure $P_{F}$ such that $P_{T, F}$ and $P_{T, \Omega, F}$ both converge weakly to $P_{F}$ as $T \rightarrow \infty$. 
Proof. Since the series for $\zeta_{n}(s, F)$ is absolutely convergent, by a standard way it followssee, for example $[14,18]$ — that the sequence $\left\{V_{n, F}: m \in \mathbb{N}\right\}$ is tight, i.e., for every $\varepsilon>0$, there exists a compact set $\left.K \subset H^{r}\left(D_{\kappa}\right)\right)$ such that

$$
V_{n, F}(K)>1-\varepsilon
$$

for all $n \in \mathbb{N}$. Hence, by the Prokhorov theorem, see [20], the sequence $\left\{V_{n, F}\right\}$ is relatively compact, i.e., each of its subsequences contains a subsequence $\left\{V_{n_{k}, F}\right\}$ such that $V_{n_{k}, F}$ converges weakly to a certain probability measure $P_{F}$ on $\left(H^{r}\left(D_{\kappa}\right), \mathcal{B}\left(H^{r}\left(D_{\kappa}\right)\right)\right)$ as $k \rightarrow \infty$.

Let $\xi_{T}$ be a random variable defined on a certain probability space with measure $v$ and uniformly distributed on $[0, T]$. Define the $H^{r}\left(D_{\kappa}\right)$-valued random element

$$
\underline{X}_{T, n, F}=\underline{X}_{T, n, F}(s)=\underline{\zeta}_{n}\left(s+i \underline{h}_{T}, F\right)
$$

and denote by $\underline{X}_{n, F}=\underline{X}_{n, F}(s)$ the $H^{r}\left(D_{\kappa}\right)$-valued random element having the distribution $V_{n, F}$. Then, by Lemma 5 , we have

$$
\underline{X}_{T, n, F} \underset{T \rightarrow \infty}{\stackrel{\mathcal{D}}{\longrightarrow}} \underline{X}_{n, F}
$$

where $\underset{T \rightarrow \infty}{\stackrel{\mathcal{D}}{\longrightarrow}}$ means the convergence in distribution. Moreover, since $V_{n_{k}, F}$ converges weakly to $P_{F}$, the relation

$$
\underline{X}_{n_{k}, F} \underset{k \rightarrow \infty}{\stackrel{\mathcal{D}}{\longrightarrow}} P_{F}
$$

is true. Let

$$
\underline{X}_{T, F}=\underline{X}_{T, F}(s)=\underline{\zeta}\left(s+i \underline{h} \underline{\xi}_{T}, F\right) .
$$

Then, using Lemma 1, we find that for every $\varepsilon>0$,

$$
\begin{aligned}
& \lim _{n \rightarrow \infty} \limsup _{T \rightarrow \infty} v\left\{\underline{\rho}\left(\underline{X}_{T, F}, \underline{X}_{T, n, F}\right) \geqslant \varepsilon\right\} \\
& \quad \leqslant \lim _{n \rightarrow \infty} \limsup _{T \rightarrow \infty} \frac{1}{\varepsilon T} \int_{0}^{T} \underline{\rho}\left(\underline{\zeta}(s+i \underline{h} \tau, F), \underline{\zeta}_{n}(s+i \underline{h} \tau, F)\right) \mathrm{d} \tau=0 .
\end{aligned}
$$

The later equality together with (8) and (9), and Theorem 4.2 of [20] lead to the relation

$$
X_{T, F} \underset{T \rightarrow \infty}{\stackrel{\mathcal{D}}{\longrightarrow}} P_{F} .
$$

This proves that $P_{T, F}$ converges weakly to $P_{F}$ as $T \rightarrow \infty$.

The relation (10) shows that the limit measure $P_{F}$ is independent of the subsequence $\left\{n_{k}\right\}$. Therefore, we have

$$
\underline{X}_{n, F} \underset{n \rightarrow \infty}{\stackrel{\mathcal{D}}{\longrightarrow}} P_{F} .
$$

Define the $H^{r}\left(D_{\kappa}\right)$-valued random elements

$$
\underline{X}_{T, n, \underline{\Omega}, F}=\underline{X}_{T, n, \underline{\Omega}, F}(s)=\underline{\zeta}_{n}\left(s+i \underline{h} \underline{\xi}_{T}, \underline{\omega}, F\right)
$$

an

$$
\underline{X}_{T, \underline{\Omega}, F}=\underline{X}_{T, \underline{\Omega}, F}(s)=\underline{\zeta}(s+i \underline{h} \tilde{\xi} T, \underline{\omega}, F) .
$$

Then, repeating the above arguments using Lemmas 2 and 5 , and relation (11), we obtain that

$$
X_{T, n, F} \underset{T \rightarrow \infty}{\stackrel{\mathcal{D}}{\longrightarrow}} P_{F}
$$

and this is equivalent to weak convergence of $P_{T, \Omega, F}$ to $P_{F}$ as $T \rightarrow \infty$. The lemma is proved. 
To prove Theorem 3 , it remains to show that $P_{F}=P_{\zeta, F}$. For this, we will apply some elements of the ergodic theory. For brevity, let

$$
\underline{h}_{\tau}=\left(\left(p^{-i h_{1} \tau}: p \in \mathbb{P}\right), \ldots,\left(p^{-i h_{r} \tau}: p \in \mathbb{P}\right)\right), \quad \tau \in \mathbb{R} .
$$

Define the transformation of $\Omega$

$$
\varphi_{\tau}(\underline{\omega})=\underline{h}_{\tau} \underline{\omega}, \underline{\omega} \in \underline{\Omega} .
$$

Since the Haar measure $\underline{m}_{H}$ is invariant, the transformation $\varphi_{\tau}$ is measure-preserving and $\left\{\varphi_{\tau}: \tau \in \mathbb{R}\right\}$ is a one-parameter group. A set $A \in \mathcal{B}(\underline{\Omega})$ is called invariant with respect to the group $\left\{\varphi_{\tau}\right\}$ if the sets $A$ and $\varphi_{\tau}(A), \tau \in \mathbb{R}$, differ one from another at most by a set of $\underline{m}_{H}$-measure zero.

Lemma 7. Let $h_{1}, \ldots, h_{r}$ be the same as Theorem 3. Then, the group $\left\{\varphi_{\tau}\right\}$ is ergodic, i.e., the $\sigma$-field of invariant sets consists of sets having $\underline{m}_{H^{-}}$measure 1 or 0 .

Proof. The characters $\chi$ of the group $\Omega$ are of the form

$$
\chi(\underline{\omega})=\prod_{j=1}^{r} \prod_{p \in \mathbb{P}}^{*} \omega_{j}^{k_{p j}}(p) .
$$

This fact already was used in the proof of Lemma 4 . Let $A$ be an arbitrary invariant set, $I_{A}$ its indicator function, and $\chi$ be a nontrivial character. Preserving the notation of the proof of Lemma 4 , we have $\left(\underline{k}_{1}, \ldots, \underline{k}_{r}\right) \neq(\underline{0}, \ldots, \underline{0})$ and $B_{\underline{k}_{1}, \ldots, \underline{k}_{r}} \neq 0$. Therefore, there exists $\tau_{0} \in \mathbb{R}$ such that

$$
\chi\left(\underline{h}_{\tau}\right)=\exp \left\{-i \tau_{0} B_{\underline{k}_{1}, \ldots, \underline{k}_{r}}\right\} \neq 1 .
$$

Moreover, in view of the invariance of $A$, we have

$$
I_{A}\left(\underline{h}_{\tau_{0}} \underline{\omega}\right)=I_{A}(\underline{\omega})
$$

for almost all $\underline{\omega} \in \underline{\Omega}$. Denote by $\hat{I}_{A}$ the Fourier transform of $I_{A}$. Then, by (13),

$$
\hat{I}_{A}(\chi)=\chi\left(\underline{h}_{\tau_{0}}\right) \int_{\underline{\Omega}} I_{A}\left(\underline{h}_{\tau_{0}} \underline{\omega}\right) \chi(\underline{\omega}) \mathrm{d} \underline{m}_{H}=\chi\left(\underline{h}_{\tau_{0}}\right) \hat{I}_{A}(\chi) .
$$

This and (12) show that

$$
\hat{I}_{A}(\chi)=0 .
$$

Now, let $\chi_{0}$ denote the trivial character of $\underline{\Omega}$, and suppose that $\hat{I}_{A}\left(\chi_{0}\right)=\alpha$. Then, in view of (14), we find that

$$
\hat{I}_{A}(\chi)=\alpha \int_{\underline{\Omega}} \chi(\underline{\omega}) \mathrm{d} \underline{m}_{H}=\hat{\alpha}(\chi)
$$

Hence, $I_{A}(\underline{\omega})=\alpha$ for almost all $\underline{\omega} \in \underline{\Omega}$. Since $I_{A}$ is the indicator function, $I_{A}(\underline{\omega})=1$ or $I_{A}(\underline{\omega})=0$ for almost all $\underline{\omega}$. Thus, $\underline{m}_{H}(A)=1$ or $\underline{m}_{H}(A)=0$, and the lemma is proved.

Proof of Theorem 3. We have mentioned that it suffices to show that $P_{F}=P_{\zeta, F}$. By Lemma 6 and the equivalent of weak convergence in terms of continuity sets, we have

$$
\lim _{T \rightarrow \infty} P_{T, \underline{\Omega}, F}(A)=P_{F}(A)
$$


for a continuity set $A$ of the measure $P_{F}$, i.e., $P_{F}(\partial A)=0$, where $\partial A$ is the boundary of $A$. On the probability space $\left(\Omega, \mathcal{B}(\underline{\Omega}), \underline{m}_{H}\right)$, define the random variable

$$
\xi(\underline{\omega})= \begin{cases}1 & \text { if } \underline{\zeta}(s, \underline{\omega}, F) \in A, \\ 0 & \text { otherwise }\end{cases}
$$

Lemma 7 implies the ergodicity of the random process $\xi\left(\varphi_{\tau}(\underline{\omega})\right)$. Therefore, by the classical Birkhoff-Khintchine ergodic theorem, see, for example [21],

$$
\lim _{T \rightarrow \infty} \frac{1}{T} \int_{0}^{T} \xi\left(\varphi_{\tau}(\underline{\omega})\right) \mathrm{d} \tau=\mathbb{E} \xi=P_{\underline{\zeta}, F}(A),
$$

where $\mathbb{E} \xi$ is the expectation of $\xi$.

However, by the definitions of $\varphi_{\tau}$ and $\xi$,

$$
\frac{1}{T} \int_{0}^{T} \xi\left(\varphi_{\tau}(\underline{\omega})\right) \mathrm{d} \tau=\frac{1}{T} \operatorname{meas}\{\tau \in[0, T]: \underline{\zeta}(s+i \underline{h} \tau, \underline{\omega}, F) \in A\}=P_{T, \underline{\Omega}, F}(A) .
$$

This and (16) show that

$$
\lim _{T \rightarrow \infty} P_{T, \underline{\Omega}, F}(A)=P_{\underline{\underline{\underline{z}}, F}}(A) .
$$

Therefore, by (15), we obtain that $P_{F}(A)=P_{\zeta, F}(A)$ for all continuity sets $A$ of $P_{F}(A)$. Hence, $P_{F}=P_{\underline{\zeta}, F}$, and the theorem is proved.

\section{Proof of Theorem 2}

Recall that the support of the measure $P_{\zeta, F}$ is a minimal closed set $S_{F} \subset H^{r}\left(D_{\kappa}\right)$ such that $P_{\underline{\zeta}, F}\left(S_{F}\right)=1$.

Lemma 8. The support of the measure $P_{\underline{\zeta}, F}$ is the set $\left(\left\{g \in H\left(D_{\mathcal{K}}\right): g(s) \neq 0 \text { or } g(s) \equiv 0\right\}\right)^{r}$.

Proof. Since the space $H^{r}\left(D_{\kappa}\right)$ is separable, we have [20],

$$
\mathcal{B}\left(H^{r}\left(D_{\kappa}\right)\right)=(\underbrace{\mathcal{B}\left(H\left(D_{\kappa}\right)\right) \times \cdots \times \mathcal{B}\left(H\left(D_{\kappa}\right)\right)}_{r} .
$$

Therefore, it suffices to consider the measure $P_{\underline{\zeta, F}}$ on the rectangular sets

$$
A=A_{1} \times \cdots \times A_{r}, \quad A_{1}, \ldots, A_{r} \in H\left(D_{\kappa}\right) .
$$

Let $\zeta(s, \omega, F)$ be the $H\left(D_{\kappa}\right)$-valued random element defined on the probability space $\left(\Omega, \mathcal{B}(\Omega), m_{H}\right)$, where $m_{H}$ is the Haar measure. Then, it is known [10] that the support of the distribution of $\zeta(s, \omega, F)$ is the set $\left\{g \in H\left(D_{\kappa}\right): g(s) \neq 0\right.$ or $\left.g(s) \equiv 0\right\}$. Thus, the same set is the support of the distributions of $\zeta\left(s, \omega_{j}, F\right), j=1, \ldots, r$. Since the measure $\underline{m}_{H}$ is the product of the measures $m_{j H}, j=1, \ldots, r$, we have

$$
\underline{m}_{H}\{\underline{\omega} \in \underline{\Omega}: \underline{\zeta}(s, \underline{\omega}, F) \in A\}=\prod_{j=1}^{r} m_{j H}\left\{\omega_{j} \in \Omega_{j}: \zeta\left(s, \omega_{j}, F\right) \in A_{j}\right\} .
$$

This equality, the minimality of the support, and the support of the distributions of $\zeta\left(s, \omega_{j}, F\right)$ prove the lemma. 
Proof of Theorem 2. By the Mergelyan theorem on the approximation of analytic functions by polynomials [22], there exist polynomials $p_{1}(s), \ldots, p_{r}(s)$ such that

$$
\sup _{1 \leq j \leq r s \in K_{j}} \sup _{1}\left|f_{j}(s)-e^{p_{j}(s)}\right|<\frac{\varepsilon}{2} .
$$

Define the set

$$
G_{\varepsilon}=\left\{\left(g_{1}, \ldots, g_{r}\right) \in H^{r}\left(D_{\kappa}\right): \sup _{1 \leq j \leq r \leq K_{j}} \sup _{s \in K_{j}}\left|g_{j}(s)-e^{p_{j}(s)}\right|<\frac{\varepsilon}{2}\right\} .
$$

In view of Lemma 8 , the set $G_{\varepsilon}$ is an open neighborhood of an element $\left(e^{p_{1}(s)}, \ldots, e^{p_{r}(s)}\right)$ in support of the measure $P_{\underline{\underline{\zeta}}, F}$. Hence,

$$
P_{\underline{\zeta}, F}\left(G_{\varepsilon}\right)>0 \text {. }
$$

This, Theorem 3 and the equivalent of weak convergence in terms of open sets, and the definitions of $P_{T, F}$ and $G_{\varepsilon}$ prove the theorem with "lim inf". Define one more set

$$
\hat{G}_{\varepsilon}=\left\{\left(g_{1}, \ldots, g_{r}\right) \in H^{r}\left(D_{\kappa}\right): \sup _{1 \leq j \leq r \leq K_{j}} \sup _{s \in}\left|g_{j}(s)-f_{j}(s)\right|<\varepsilon\right\},
$$

There $\partial \hat{G}_{\varepsilon_{1}} \cap \partial \hat{G}_{\varepsilon_{2}}=\varnothing$ for $\varepsilon_{1} \neq \varepsilon_{2}$. This shows that $P_{\underline{\underline{\zeta}}, F}\left(\partial \hat{G}_{\varepsilon}\right)=0$ for all but, for those countable, many $\varepsilon>0$. Moreover, (17) and (18) imply that $P_{\zeta, F}\left(\hat{G}_{\varepsilon}\right)>0$. This, Theorem 3 and the equivalent of weak convergence of probability measures in terms of continuity sets, and the definitions of $P_{T, F}$ and $\hat{G}_{\varepsilon}$ prove the theorem with "lim".

Funding: This research received no external funding.

Conflicts of Interest: The author declares no conflict of interest.

\section{References}

1. Voronin, S.M. Theorem on the "universality" of the Riemann zeta-function. Math. USSR Izv. 1975, 9, 443-453. [CrossRef]

2. Laurinčikas, A.; Matsumoto, K. The universality of zeta-functions attached to certain cusp forms. Acta Arith. 2001, 98, 345-359. [CrossRef]

3. Laurinčikas, A.; Matsumoto, K.; Steuding, J. Discrete universality of L-functions for new forms. Math. Notes 2005, 78, 551-558. [CrossRef]

4. Laurinčikas, A.; Matsumoto, K.; Steuding, J. Discrete universality of L-functions for new forms. II. Lith. Math. J. 2016, 56, 207-218. [CrossRef]

5. Laurinčikas, A.; Matsumoto, K.; Steuding, J. Universality of some functions related to zeta-functions of certain cusp forms. Osaka J. Math. 2013, 50, 1021-1037.

6. Laurinčikas, A. On the functional independence of zeta-functions of certain cusp forms. Math. Notes 2020, 107, 609-617. [CrossRef]

7. Laurinčikas, A.; Matsumoto, K.; Steuding, J. The universality of L-functions associated with new forms. Izv. Math. 2003, 67, 77-90. [CrossRef]

8. Vaiginytè, A. Extention of the Laurinčikas-Matsumoto theorem. Chebyshevskì Sb. 2019, 20, 82-93.

9. Laurinčikas, A.; Šiaučiūnas, D.; Vaiginytè, A. Extension of the discrete universality theorem for zeta-functions of certain cusp forms. Nonlinear Anal. Model. Control 2018, 23, 961-973. [CrossRef]

10. Balčiūnas, A.; Franckevič, V.; Garbaliauskienè, V.; Macaitienè, R.; Rimkevičienè, A. Universality of zeta-functions of cusp forms and non-trivial zeros of the Riemann zeta-function. Math. Model. Anal. 2021, 26, 82-93. [CrossRef]

11. Voronin, S.M. On the functional independence of Dirichlet $L$-functions. Acta Arith. 1975, 27, 493-503. (In Russian)

12. Laurinčikas, A.; Matsumoto, K. The joint universality of twisted automorphic L-functions. J. Math. Soc. Jpn. 2004, 56, 923-939. [CrossRef]

13. Laurinčikas, A.; Šiaučiūnas, D., Vaiginytè, A. On joint approximation of analytic functions by nonlinear shifts of zeta-functions of certain cusp forms. Nonlin. Anal. Model. Control 2020, 25, 108-125. [CrossRef]

14. Laurinčikas, A. Joint universality of zeta-functions with periodic coefficients. Izv. Math. 2010, 74, 515-539. [CrossRef]

15. Laurinčikas, A. Joint universality of zeta functions with periodic coefficients. II. Sib. Math. J. 2020, 61, 1064-1076. [CrossRef]

16. Laurinčikas, A. Joint discrete universality for periodic zeta-functions. III. Quaest. Math. 2020. [CrossRef] 
17. Kaczorowski, J.; Laurinčikas, A.; Steuding, J. On the value distribution of shifts of universal Dirichlet series. Monatsh. Math. 2006, 147, 309-317. [CrossRef]

18. Kačènas, A.; Laurinčikas, A. On Dirichlet series related to certain cusp forms. Lith. Math. J. 1998, 38, 64-76. [CrossRef]

19. Baker, A. The theory of linear forms in logarithms. In Transcendence Theory: Advances and Applications. Proceedings of a Conference Held in Cambridge in 1976; Baker, A., Masser, D.W., Eds.; Academic Press: Boston, MA, USA, 1977; pp. 1-27.

20. Billingsley, P. Convergence of Probability Measures; Willey: New York, NY, USA, 1968.

21. Cramér, H.; Leadbetter, M.R. Stationary and Related Stochastic Processes; Willey: New York, NY, USA, 1967.

22. Mergelyan, S.N. Uniform approximations to functions of a complex variable. Am. Math. Soc. Transl. Ser. 2 1962, 3, $294-391$. 